\title{
An Efficient Link Prediction Technique in Social Networks based on Node Neighborhoods
}

\author{
Gypsy Nandi \\ Assam Don Bosco University \\ Guwahati, Assam, India
}

\author{
Anjan Das \\ St. Anthony's College \\ Shillong, Meghalaya, India
}

\begin{abstract}
The unparalleled accomplishment of social networking sites, such as Facebook, LinkedIn and Twitter has modernized and transformed the way people communicate to each other. Nowadays, a huge amount of information is being shared by online users through these social networking sites. Various online friendship sites such as Facebook and Orkut, allow online friends to share their thoughts or opinions, comment on others' timeline or photos, and most importantly, meet new online friends who were known to them before. However, the question remains as to how to quickly propagate one's online network by including more and more new friends. For this, one of the easy methods used is list of 'Suggested Friends' provided by these online social networking sites. For suggestion of friends, prediction of links for each online user is needed to be made based on studying the structural properties of the network. Link prediction is one of the key research directions in social network analysis which has attracted much attention in recent years. This paper discusses about a novel efficient link prediction technique LinkGyp and many other commonly used existing prediction techniques for suggestion of friends to online users of a social network and also carries out experimental evaluations to make a comparative analysis among each technique. Our results on three real social network datasets show that the novel LinkGyp link prediction technique yields more accurate results than several existing link prediction techniques.
\end{abstract}

Keywords-Link prediction; online social networks; common neighbors; Jaccard's coefficient; Adamic/Adar; preferential attachment; FriendLink

\section{INTRODUCTION}

Online Social Networks (OSNs) have become a means for millions of online users to express and share their opinions with other users. These OSNs provide an excellent opportunity for allowing interactions and exchange of thoughts, opinions and ideas among the online users in a group or community. Such networks can be represented as graphs, where a node or a vertex corresponds to a user present in the graph and an edge corresponds to any form of association between the nodes or users, such as friendship ties. Also, these OSNs are dynamic and this raises a question as to: how does the graph structure of these networks change over time? Based on this question, this paper studies about the likeliness of any two nodes of a graph to be associated in the near future, considering that presently there is no connection in the current snapshot of the OSN graph being studied. This problem, commonly called the link prediction problem, is a research area being studied by many researchers in this field to generate faster and more appropriate result with special consideration to scalability and dynamic nature of the graph. Fig. 1 gives a basic idea about how link prediction is done by studying the structural links of a network. In this figure, five nodes have been considered at time $t$ and future predictions are being made at time $t+1$. By studying the existing links, two predictions are made which are marked as dashed lines in the figure.

Liben-Nowell and Kleinberg [1] were the first to study the link prediction problem and propose a prediction model for the same. Their model mainly studies the linkage structure of a social network and discusses several link prediction methods for inferring new links. In [2], Hasan et al. studied several classification models for possible link prediction in coauthorship domain that aimed to provide a comparison of several features using different feature analysis algorithms. Zheleva et al. [3] studied binary classification algorithm that mainly studies friend or family networks for link prediction. They have mainly worked on the predictive power of overlaying friendship and family ties on three real world social networks. In [4], Tylenda et al. studied time-aware and timeagnostic maximum entropy methods in which time-based weighting of edges were used. Chen et al. [5] made a detailed study and comparison of four algorithms related to link prediction, namely, Friend of a Friend (FOAF), SONAR, Content-plus-Link (CplusL) and content matching algorithms. Schifanella et al. [6] considered a sampling link prediction algorithm that can help users find friends with similar topical interests as well as facilitate the formation of topical communities. They also introduced a null model to show that a part of the similarity between online users is due to the correlations between user activity and user degree centrality in the OSN. In [7], Papadimi-triou et al. found the FriendLink Algorithm for fast and accurate link prediction in OSNs which outperforms many other related algorithms in terms of accuracy and time-complexity. Bayesian network has been also considered as a consistent model to understand the relations between future links to be predicted in networks [19], [20]. Recently, negative link prediction in social networks has attracted the attention of many researchers and considerable research work is being carried out to find efficient techniques for the same [15], [16], [18]. Such techniques aim to perform link prediction across multiple signed networks. Recent work has also focused on noise-filtering technique to predict links in complex networks [17]. 


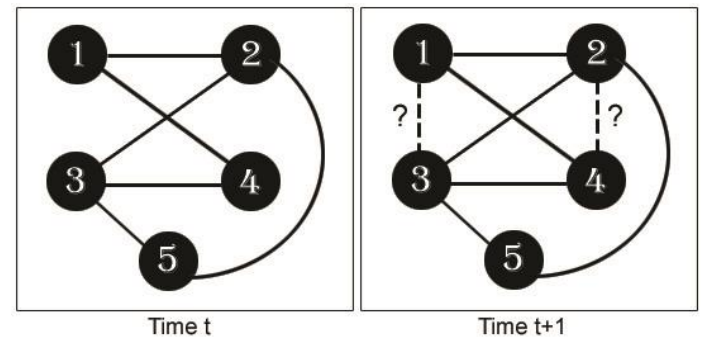

Fig. 1. Example of prediction of links in a given network.

The rest of the paper is organized as follows. In Section 2, a discussion on five standard link prediction techniques, namely, the Common Neighbors, Jaccard's coefficient, Adamic/Adar, Preferential Attachment, and FriendLink are given. Section 3 discusses about a novel link prediction technique LinkGyp which aims to provide better result than the above mentioned existing link prediction techniques. Section 4 illustrates the experimental results by comparing the various predictions of links made by the existing techniques with the novel technique. A conclusion of the paper and discussion on the scope for future work is given in Section 5.

\section{DISCUSSION ON STANDARD LINK PREDICTION TECHNIQUES}

In this section, we at first discuss five standard link prediction techniques used in social networks and give a comparative analysis of the same. All these five techniques use the local-based features of a graph. There are, however, many global-based approaches also, which utilize the entire path structure in a network, but such approaches are computationally expensive for even a decent-sized social networks.

As seen in Fig. 1, vertices 1-5 indicates that there are five nodes or users in the network and the edges represent the existing links between each two nodes. In all the techniques explained below, an OSN is considered which is modeled as an directed or undirected graph $G=(V, E)$, where $V$ denotes a set of vertices and $E$ denotes a set of edges between two vertices in the network. Given below are some simple local similarity approaches based on node neighborhoods.

\section{A. Common Neighbors}

The technique of finding common neighbors for link prediction is considered as the most basic and significant method for prediction of links among nodes in the network. This approach was initially applied in the context of collaboration networks by Newman [8]. The basic idea of this technique is to find out the number of common nodes or neighbors or friends between two non-neighbors or nonfriends. Now, the higher the number of common neighbors, the more likely is the chances of those two non-neighbors of being linked in the near future.

Using this concept, a link prediction score can be calculated between any two nodes $\mathrm{p}$ and $\mathrm{q}$, where $\mathrm{p}$ and $\mathrm{q}$ are nonneighbors or non-friends at a given time $t$. The probability that these two nodes $\mathrm{p}$ and $\mathrm{q}$ will be linked in the near future is based on the score value given below:

$$
\operatorname{score}(p, q)=\operatorname{Neighbors}(p) \cap \operatorname{Neighbors}(q)
$$

Considering Fig. 1, studying the network at time $t$, a prediction of future links (at time $t+1$ ) can be made between nodes 1 and 3 as well as 2 and 4 . This is so because the number of common neighbors or the score value in both the cases is 2 (which are higher compared to the rest of the non-neighbors in the network). The technique of common neighbors is very simple and easy to analyze; yet this technique is very effective and it has been experimentally evaluated that it often outperforms several other complicated techniques used for link prediction. Algorithm 1 explains the link prediction technique based on the concept of common neighbors scores. In this algorithm, the social network $G$ and the top ' $n$ ' link predictions are taken as input and the top ' $n$ ' nodes based on the score value is displayed as output. The score is calculated for each non-neighbors of a node and top- $n$ predictions for the node are made based on the descending order of score values.

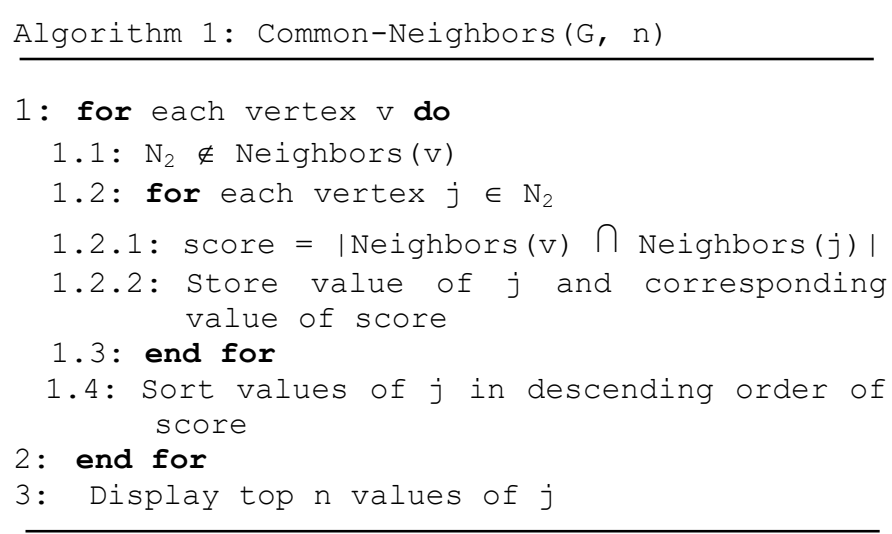

\section{B. Jaccard's Coefficient}

Jaccard's coefficient [9] is another simple technique of link prediction which is similar to common neighbors' technique discussed above as this technique also relies on the number of common neighbors between two nodes. In case of Jaccard's coefficient, the probability that two nodes $p$ and $q$ will be linked in the near future is based on the score value given below:

$$
\operatorname{score}(p, q)=\frac{|\operatorname{Neighbors}(p) \cap \operatorname{Neighbors}(q)|}{|\operatorname{Neighbors}(p) \cup \operatorname{Neighbors}(q)|}
$$

As can been seen from the score calculation mentioned above, in case of Jaccard's coefficient, the number of common neighbors is simply divided by the number of total neighbors. For instance, in Fig. 1, the score of nodes 1 and 3 at time t, using Jaccard's coefficient of link estimation is 0.67. Similarly, score of nodes 1 and 5 at time $t$ is 0.33 . Hence, there is a higher chance of nodes 1 and 3 being linked in the near future compared to nodes 1 and 5 . Hence, the score value between two nodes will always remain between 0 and 1; 0 when there is no single common neighbor between two nodes and 1 when the two nodes being compared is the same node. Algorithm 2 explains the link prediction technique based on the concept of Jaccard Coefficient scores. In this algorithm, the social network $\mathrm{G}$ and the top ' $n$ ' link predictions are taken as input and the top ' $n$ ' nodes base on the score value is displayed as output. 
Algorithm 2: Jaccard-Coefficient (G, n)

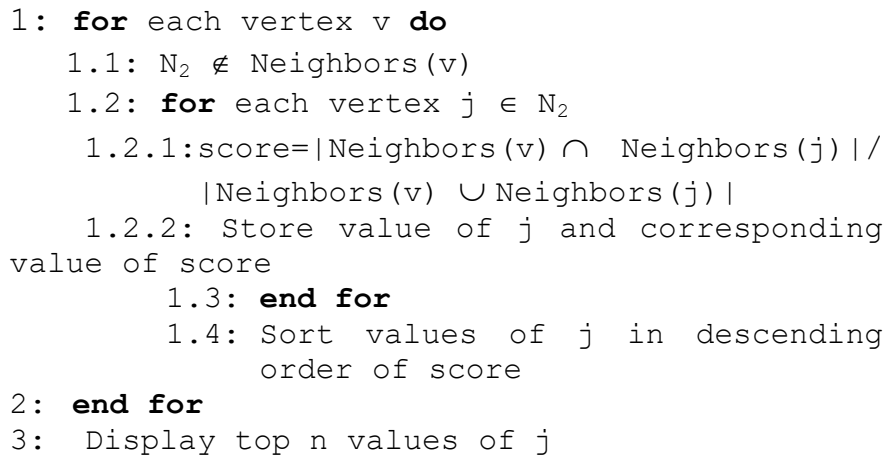

\section{Adamic/Adar}

Adamic and Adar [10] have found another approach to predict links between two nodes in a network. In Adamic/Adar technique, all the common neighbors of two non-friends or non-neighbors are taken into consideration, and how many connections each of these common neighbors have are also considered. Thus, the probability that two nodes $\mathrm{p}$ and $\mathrm{q}$ will be linked in the near future is based on the score value given below:

$$
\operatorname{score}(p, q)=\sum_{x \in N(p) \cap N(q)} \frac{1}{\log |\operatorname{Neighbors}(x)|}
$$

Here $\mathrm{x}$ represents the set of common neighbors of nodes $p$ and $q$ and in the score calculation the number of neighbors of $\mathrm{x}$ is taken into consideration. In such a case, if a neighbor of $p$ and $q$ has two links or friends, a weight of $1 / \log (2)=1.4$ is considered. And again, if a neighbor of $p$ and $q$ has five links, a weight of $1 / \log (5)=0.62$ is considered. Hence, the more links a neighbor has, a better score value is obtained. Algorithm 3 explains the link prediction technique based on the concept of Adamic/Adar scores. In this algorithm, the social network $\mathrm{G}$ and the top ' $n$ ' link predictions are taken as input and the top ' $n$ ' nodes based on the score values is displayed as output.

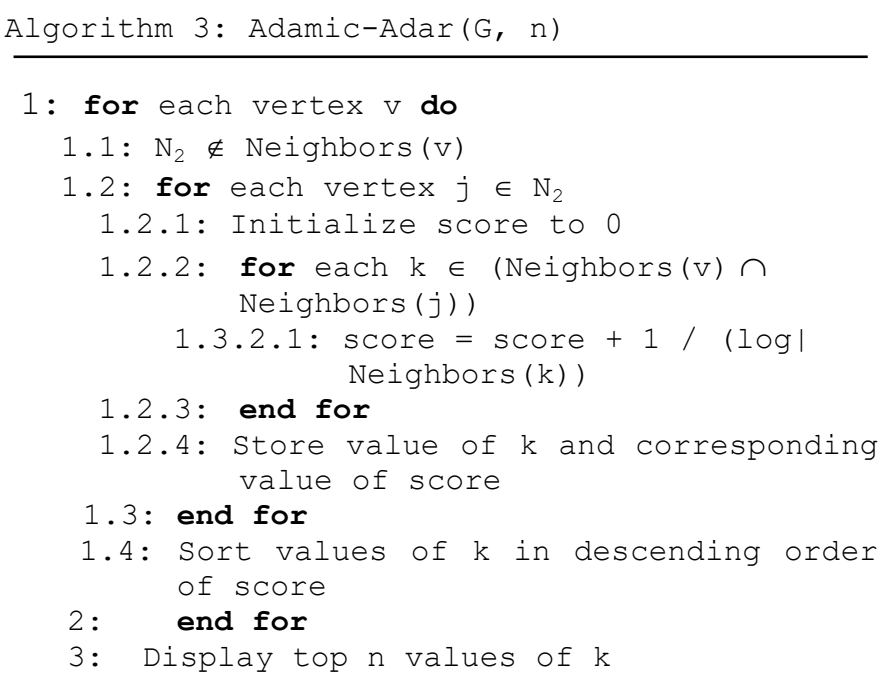

\section{Preferential Attachment}

The technique of Preferential Attachment for predicting links in a network is based on the concept that two nonneighbors or non-friends have higher chances of being connected by a link in the future if the product of their number of individual neighbors is high. This results in the calculation of score value as given below:

$$
\operatorname{score}(p, q)=\operatorname{Neighbors}(p) . \operatorname{Neighbors}(q)
$$

The term preferential attachment refers to the observation that in networks that grow over time, the likelihood that an edge is added to a node with $n$ neighbors is proportional to $n$ [11]. Experiments conducted by researchers have revealed that co-authorship is correlated with the product of the neighborhood sizes and the similar concept is applied for link prediction in social networks. Algorithm 4 explains the link prediction technique based on the concept of Preferential Attachment scores. In this algorithm, the social network $G$ and the top ' $n$ ' link predictions are taken as input and the top ' $n$ ' nodes based on the score values is displayed as output.

Algorithm 4: Preferential-Attachment ( $G, n$ )

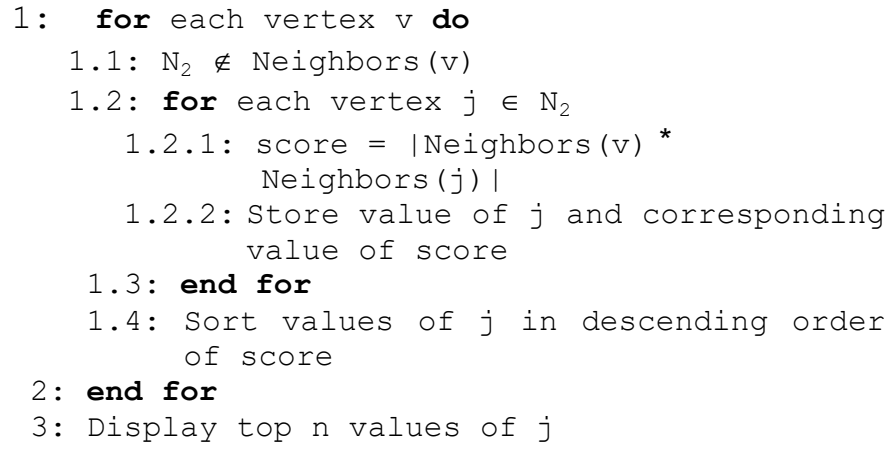

\section{E. FriendLink}

The FriendLink [7] approach of link prediction studies user's neighborhood by making use of paths of greater length. Here, two users connected with many unique pathways have a higher likelihood to know each other. Algorithm 5 explains the FriendLink approach for link prediction which takes as input the social graph $G$, the adjacency matrix $A$ of graph $G$, number of nodes ' $n$ ' present in the graph, and the maximum length of paths ' $l$ ' explored in $G$. The algorithm provides as output the similarity matrix between two nodes in $G$. Based on the weights of similarity matrix friends can be recommended for a target node.

In the main program of Algorithm 5, the adjacency matrix of the graph is modified so that instead of holding the traditional values 0 or 1 , the matrix is filled up with values 0 or $\mathrm{vj}$, where $\mathrm{vj}$ is a node to which node vi is connected. In the function ComputePaths(), matrix multiplication of this modified adjacency matrix is performed with itself to produce all paths from node $v_{i}$ to node $v_{j}$. Lastly, in the function ComputeSimilarity(), the similarity value between two nodes is measured to estimate the strength of connections between two non-linked nodes. 
no improvement in the result. Hence giving a right input value of $\mathrm{n}(5 \leq n \leq 50)$ will lead to better and more accurate results. The rest of the other non-edged nodes are discarded and further steps are carried out only for the top $2 n$ resulting nodes. These few steps are carried out keeping in mind the ground truth in economics that "the rich get richer". Also, it results in a smaller choice of nodes with least computational complexity.

Next, a similar approach to Adamic/Adar explained above is followed to find the ' $n$ ' best predicted non-edged nodes for vertex ' $\mathrm{v}$ ' from the set of only $2 n$ number of nodes selected in steps 1.1 to 1.4 . The reason behind choosing the Adamic/Adar approach is that this technique considers the case that an affair owned by less objects, compared to owned by more objects, has greater effect on link prediction. In this way, the scores are calculated for each of the two non-edged nodes and ultimately the output for top- $n$ predicted nodes is displayed for each unique user $\mathrm{v}$ based on the descending order of score.

The idea behind using this algorithm is mainly the scalability issue while dealing with dense social networks. As mentioned before, from steps 1.6 to 1.10 , the estimation of links is done for a very small subgraph consisting of only $2 n$ nodes, where ' $n$ ' is the number of prediction of links to be made. Prior to step 1.5, the calculation of score 1 is simple and does not involve studying in-depth the entire social network. It is only basically studying how many neighbors two non-edged nodes have. Hence, this algorithm proves to be an efficient method of prediction of links in social networks.

\section{Complexity Analysis of the LinkGyp Algorithm}

Online social networks are usually largely populated with information. Link prediction algorithms based on global based features, such as Katz index or Random Walk with Restart, are computationally too expensive for large graphs as it involves the inversion of matrix for link prediction. However, the standard existing link prediction algorithms discussed above are based on local based features, and comparatively have less time complexity than global based feature algorithms.

If we specifically consider the time complexity of our proposed LinkGyp technique, it is mainly $\mathrm{O}(2 \mathrm{n})$, where ' $n$ ' is the number of link predictions to be made per node. This is much effective in terms of complexity analysis as the value of ' $n$ ' will be significantly much smaller compared to the total number of nodes ' $g$ ' for the entire graph. However, most of the other discussed link prediction techniques (such as, Jaccard Coefficient, Adamic/Adar, etc.) consider the entire nodes of the graph for prediction of links for a particular node that in realtime would be in terms of thousands, lakhs or even more. Hence, the complexity of Jaccard coefficient and Adamic/Adar techniques is $\mathrm{O}(\mathrm{g})$, where the value of ' $g$ ' is significantly greater than ' $n$ '. For Friendlink algorithm, the time complexity is $\mathrm{O}(\mathrm{g} \times \mathrm{al})$, where ' $a$ ' is the average nodes degree in a graph and ' $l$ ' refers to the path lengths. Thus, the basic idea of LinkGyp algorithm is that the estimation of links is done for a very small subgraph consisting of only $2 n$ nodes, which gives better results as far as complexity of time is to be considered. The next section discusses the experimental results which prove that the above discussed novel link prediction algorithm gives a considerably better output compared to several basic existing link prediction techniques.

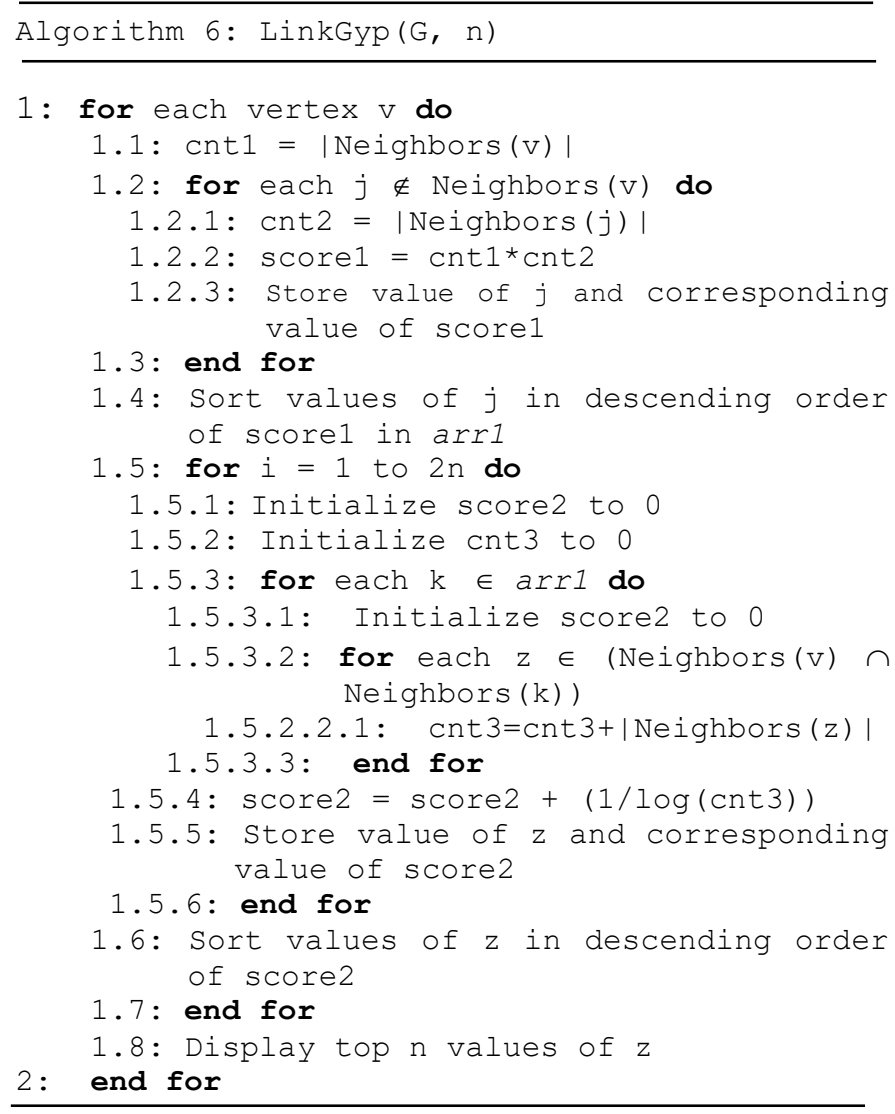

\section{EXPERIMENTAL EVALUATION}

For conducting the experiments, three publicly available real-world datasets have been used that contains friendship network between users of social networking websites, namely the facebook dataset [24], the hamsterster dataset [12] and the brightkite location-based social networking website [21]. Table I gives few statistical information of all the three datasets.

TABLE I. StATistics OF THE VARIOUS DATASETS

\begin{tabular}{|l|l|l|l|}
\hline Dataset & facebook & hamsterster & brightkite \\
\hline \#Nodes & 63731 & 1858 & 55228 \\
\hline \#Edges & 817035 & 12534 & 214078 \\
\hline $\begin{array}{l}\text { Average } \\
\text { Degree }\end{array}$ & 25.640 & 13.492 & 7.353 \\
\hline $\begin{array}{l}\text { Maximal } \\
\text { Degree }\end{array}$ & 1098 & 272 & 272 \\
\hline $\begin{array}{l}\text { Average Path } \\
\text { Length }\end{array}$ & 2.832 & 3.453 & 2.76 \\
\hline
\end{tabular}

a) The facebook dataset is an undirected network containing 63,731 nodes and 817035 edges that describes friendship data of facebook users. A node represents a user and an edge represents a friendship between two users. Fig. 2 illustrates the graphical view of the facebook dataset (nodes having degree less than six have not been considered) As can be seen from the figure, the yellow colored, bigger-sized nodes have highest degrees, the blue colored moderate-sized nodes have average degrees and red colored small-sized nodes have fewer degrees. 


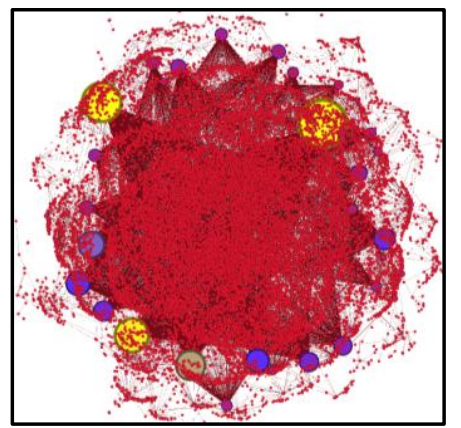

Fig. 2. The facebook dataset represented as a graph having different sized and colored nodes based on degree.

b) The hamsterster friendship dataset contains 1858 distinct nodes and 12534 edges which indicates the ties or friendship among all users in the network. The entire dataset has been represented in a graph as shown in Fig. 3 in which the yellow colored, bigger-sized nodes have highest degrees, the blue colored moderate-sized nodes have average degrees and red colored small-sized nodes have fewer degrees.

c) The brightkite friendship dataset contains undirected user-user friendship relations that have been gathered from a former widely used location-based social network. This dataset contains 55,228 distinct nodes and 214,078 edges that indicate friendship ties between two users. Fig. 4 illustrates the graphical form of a slice of the brightkite dataset (nodes having degree of one have not been considered) in which, the yellow colored, bigger-sized nodes have highest degrees, the blue colored moderate-sized nodes have average degrees and red colored small-sized nodes have fewer degrees.

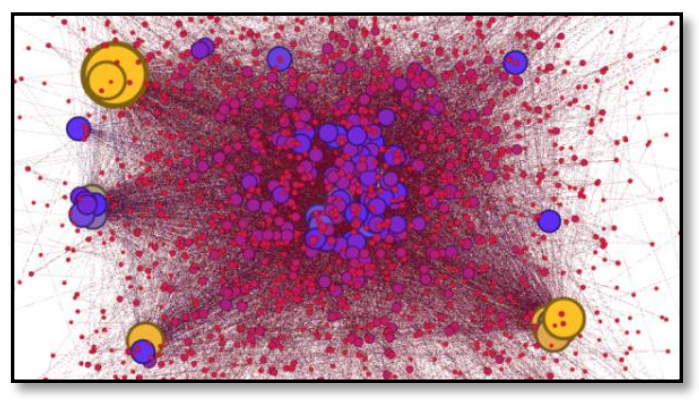

Fig. 3. The hamsterster dataset represented as a graph having different sized and colored nodes based on degree.

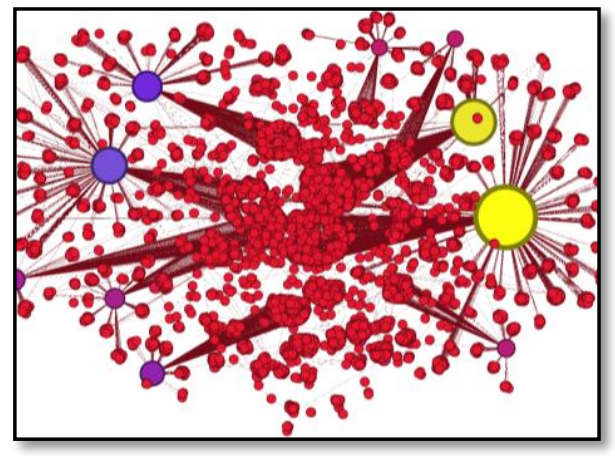

Fig. 4. The brightkite dataset represented as a graph having different sized and colored nodes based on degree.
Several experiments were conducted for the link prediction techniques mentioned above on all the three datasets. These experiments mainly aim at illustrating the performance comparison of the above mentioned link prediction techniques when compared to the random method generation of links for predicting future associations among nodes.

To conduct all the experiments, each of the entire dataset was divided into training and testing datasets consisting of $60 \%$ and $40 \%$ records respectively. Care was taken to include at least all core nodes in the training data set ('core' is the set containing nodes they have a direct link to minimum 10 other nodes). Tables II to VII illustrate the number of common predictions made between each two techniques for the two datasets which basically demonstrates which techniques are similar to each other in generation of link prediction results.

From the results obtained from Tables II to VII, it can be concluded that the random generation technique yields the least common predictions compared to the other six link prediction techniques. It can also be considered from all these tables that Common Neighbors and Jaccard's Coefficient predicts more similar friend suggestions for future links compared to the rest of the link prediction techniques.

TABLE II. THE NUMBER OF COMMON PREDICTIONS MADE ON THE FACEBOOK DATASET OUT OF 10000 (1000 USERS X 10) PREDICTIONS

\begin{tabular}{|c|c|c|c|c|c|c|c|}
\hline & 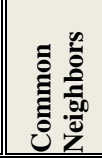 & 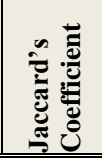 & 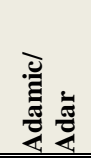 & 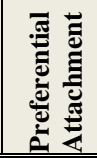 & 兰 & 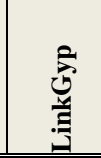 & ह气 \\
\hline $\begin{array}{l}\text { Common } \\
\text { Neighbors }\end{array}$ & 10000 & 8275 & 2504 & 2486 & 8175 & 7442 & 167 \\
\hline $\begin{array}{l}\text { Jaccard's } \\
\text { Coefficient }\end{array}$ & & 0000 & 1859 & 2144 & 6879 & 3301 & 173 \\
\hline $\begin{array}{l}\text { Adamic/ } \\
\text { Adar }\end{array}$ & & & 10000 & 2570 & 4269 & 4957 & 204 \\
\hline $\begin{array}{l}\text { Preferential } \\
\text { Attachment }\end{array}$ & & & & 10000 & 3578 & 8146 & 196 \\
\hline FriendLink & & & & & 10000 & 7686 & 185 \\
\hline LinkGyp & & & & & & 10000 & 168 \\
\hline $\begin{array}{l}\text { Random } \\
\text { Generation }\end{array}$ & & & & & & & 10000 \\
\hline
\end{tabular}

TABLE III. THE NUMBER OF COMMON PREDICTIONS MADE ON THE FACEBOOK DATASET OUT OF 20000 (1000 USERS X 20) PREDICTIONS

\begin{tabular}{|c|c|c|c|c|c|c|c|}
\hline & 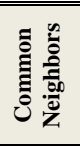 & 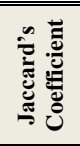 & 旁 & 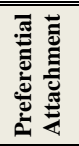 & 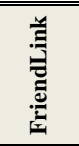 & 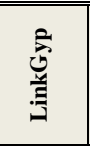 & 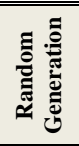 \\
\hline $\begin{array}{c}\text { Common } \\
\text { Neighbors }\end{array}$ & 20000 & 17751 & 6687 & 4591 & 15239 & 6157 & 427 \\
\hline $\begin{array}{c}\text { Jaccard's } \\
\text { Coefficient }\end{array}$ & & 20000 & 5826 & 4044 & 13486 & 6312 & 394 \\
\hline $\begin{array}{c}\text { Adamic/ } \\
\text { Adar }\end{array}$ & & & 20000 & 3746 & 10017 & 7916 & 267 \\
\hline $\begin{array}{l}\text { Preferential } \\
\text { Attachment }\end{array}$ & & & & 20000 & 9810 & 16984 & 205 \\
\hline FriendLink & & & & & 20000 & 14012 & 196 \\
\hline LinkGyp & & & & & & 20000 & 278 \\
\hline $\begin{array}{c}\text { Random } \\
\text { Generation }\end{array}$ & & & & & & & 20000 \\
\hline
\end{tabular}


TABLE IV. THE NUMBER OF COMMON PREDICTIONS MADE ON THE HAMSTERSTER DATASET OUT OF 18580 (1858 USERS X 10) PREDICTIONS

\begin{tabular}{|c|c|c|c|c|c|c|c|}
\hline & 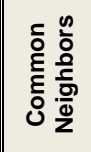 & 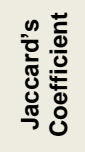 & 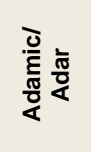 & 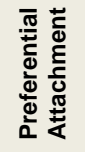 & 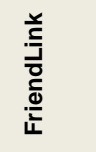 & 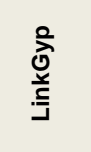 & 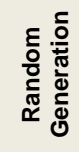 \\
\hline $\begin{array}{l}\text { Common } \\
\text { Neighbors }\end{array}$ & 18580 & 14833 & 5758 & 4218 & 16891 & 2983 & 208 \\
\hline $\begin{array}{l}\text { Jaccard's } \\
\text { Coefficient }\end{array}$ & & 18580 & 3384 & 2991 & 13567 & 1260 & 85 \\
\hline $\begin{array}{c}\text { Adamic/ } \\
\text { Adar }\end{array}$ & & & 18580 & 3710 & 13001 & 3258 & 131 \\
\hline $\begin{array}{l}\text { Preferential } \\
\text { Attachment }\end{array}$ & & & & 18580 & 11763 & 13728 & 169 \\
\hline FriendLink & & & & & 18580 & 12023 & 148 \\
\hline LinkGyp & & & & & & 18580 & 133 \\
\hline $\begin{array}{c}\text { Random } \\
\text { Generation }\end{array}$ & & & & & & & 18580 \\
\hline
\end{tabular}

TABLE V. THE Number of COMMON PREDICTIONS MADE ON THE HAMSTERSTER DATASET OUT OF 37160 (1858 USERS X 20) PREDICTIONS

\begin{tabular}{|c|c|c|c|c|c|c|c|}
\hline & 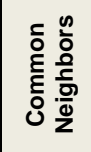 & 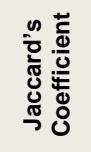 & $\begin{array}{l}\frac{\overline{0}}{\bar{E}} \\
\frac{\pi}{0} \frac{\pi}{\alpha}\end{array}$ & 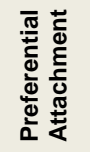 & 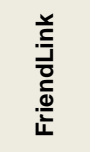 & 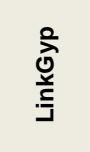 & 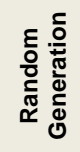 \\
\hline $\begin{array}{c}\text { Common } \\
\text { Neighbors }\end{array}$ & 31760 & 27940 & 13572 & 5589 & 27438 & 6413 & 444 \\
\hline $\begin{array}{l}\text { Jaccard's } \\
\text { Coefficient } \\
\end{array}$ & & 31760 & 9154 & 3093 & 24890 & 3018 & 360 \\
\hline $\begin{array}{c}\text { Adamic/ } \\
\text { Adar }\end{array}$ & & & 31760 & 5122 & 22769 & 7200 & 285 \\
\hline $\begin{array}{l}\text { Preferential } \\
\text { Attachment }\end{array}$ & & & & 31760 & 20026 & 26728 & 294 \\
\hline FriendLink & & & & & 31760 & 24374 & 251 \\
\hline LinkGyp & & & & & & 31760 & 263 \\
\hline $\begin{array}{c}\text { Random } \\
\text { Generation }\end{array}$ & & & & & & & 31760 \\
\hline
\end{tabular}

TABLE VI. THE Number of COMMON PREDICTIONS MADE ON THE BRIGHTKITE DATASET OUT OF 20000 (10000 X 10) PREDICTIONS

\begin{tabular}{|c|c|c|c|c|c|c|c|}
\hline & 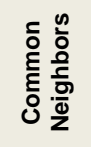 & 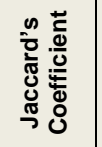 & 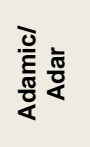 & 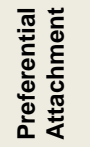 & 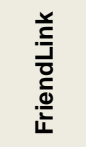 & 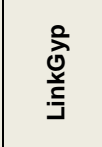 & 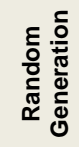 \\
\hline $\begin{array}{l}\text { Common } \\
\text { Neighbors }\end{array}$ & 20000 & 13076 & 9394 & 3186 & 10782 & 4280 & 176 \\
\hline $\begin{array}{l}\text { Jaccard's } \\
\text { Coefficient }\end{array}$ & & 20000 & 6702 & 1090 & 8201 & 2132 & 154 \\
\hline Adamic/ Adar & & & 20000 & 2080 & 7658 & 2824 & 168 \\
\hline $\begin{array}{l}\text { Preferential } \\
\text { Attachment }\end{array}$ & & & & 20000 & 7105 & $162 \mathrm{~s} 62$ & 186 \\
\hline FriendLink & & & & & 20000 & 15396 & 154 \\
\hline LinkGyp & & & & & & 20000 & 202 \\
\hline $\begin{array}{c}\text { Random } \\
\text { Generation }\end{array}$ & & & & & & & 20000 \\
\hline
\end{tabular}

TABLE VII. THE NUMBER OF COMMON PREDICTIONS MADE ON THE BRIGHTKITE DATASET OUT OF 40000 (10000 X 20) PREDICTIONS

\begin{tabular}{|c|c|c|c|c|c|c|c|}
\hline & 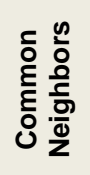 & 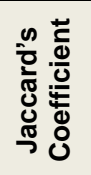 & $\frac{\bar{d}}{\frac{0}{\frac{0}{0}}} \frac{\overline{0}}{\frac{0}{0}}$ & 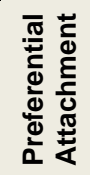 & 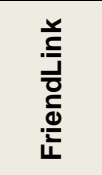 & 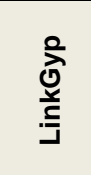 & 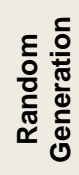 \\
\hline $\begin{array}{l}\text { Common } \\
\text { Neighbors }\end{array}$ & 40000 & 31750 & 19206 & 6236 & 28106 & 8872 & 184 \\
\hline $\begin{array}{l}\text { Jaccard's } \\
\text { Coefficient }\end{array}$ & & 40000 & 17544 & 3844 & 25871 & 7608 & 256 \\
\hline $\begin{array}{l}\text { Adamic/ } \\
\text { Adar }\end{array}$ & & & 40000 & 5818 & 24712 & 12512 & 280 \\
\hline $\begin{array}{l}\text { Preferential } \\
\text { Attachment }\end{array}$ & & & & 40000 & 23108 & 26792 & 358 \\
\hline FriendLink & & & & & 40000 & 27115 & 298 \\
\hline LinkGyp & & & & & & 40000 & 407 \\
\hline $\begin{array}{c}\text { Random } \\
\text { Generation }\end{array}$ & & & & & & & 40000 \\
\hline
\end{tabular}

Experiments were also conducted to find the number of correct predictions made on the testing datasets so as to find which techniques yield better results. A result of this is depicted in the Fig. 5-7 which again compare the above mentioned link prediction techniques against the novel LinkGyp link prediction technique for all the three datasets. Considerations were made for values of ' $n$ ' as 10 and 20, where ' $n$ ' is the number of predictions to be made for a particular node. Link predictions, in turn, were made for 1000 random distinct nodes present in the facebook dataset, and for each of the 1858 distinct nodes present in the hamsterster dataset, as well as for 2000 distinct nodes present in the brightkite dataset. Experiments reveal that the novel LinkGyp technique yields more accurate results followed by the FiendLink (considering lengths of path 2), Preferential Attachment and Adamic/Adar techniques. However, the random generation technique of link prediction, which randomly chooses the ' $n$ ' non-friends of a node, fails to come at par with all the other five prediction techniques.

The hamsterster dataset consists of densely-edged connections compared to the other dataset taken into consideration, namely the facebook dataset and the brightkite dataset which consists of comparatively sparsely-edged connections. Hence, it can be concluded that the novel LinkGyp link prediction technique can be considered as an efficient technique for link prediction keeping in mind the performance, scalability and execution time while dealing with social networks that comprise of thousands, lakhs or even more unique users and this technique is suitable for both denselyedged and sparsely-edged connections.

In summary, the results displayed in Fig. 5-7 indicate that results might slightly differ based on the scalability and sparseness of the dataset we are working upon. However, our novel LinkGyp technique outperforms other mentioned link prediction techniques in terms of accuracy. 


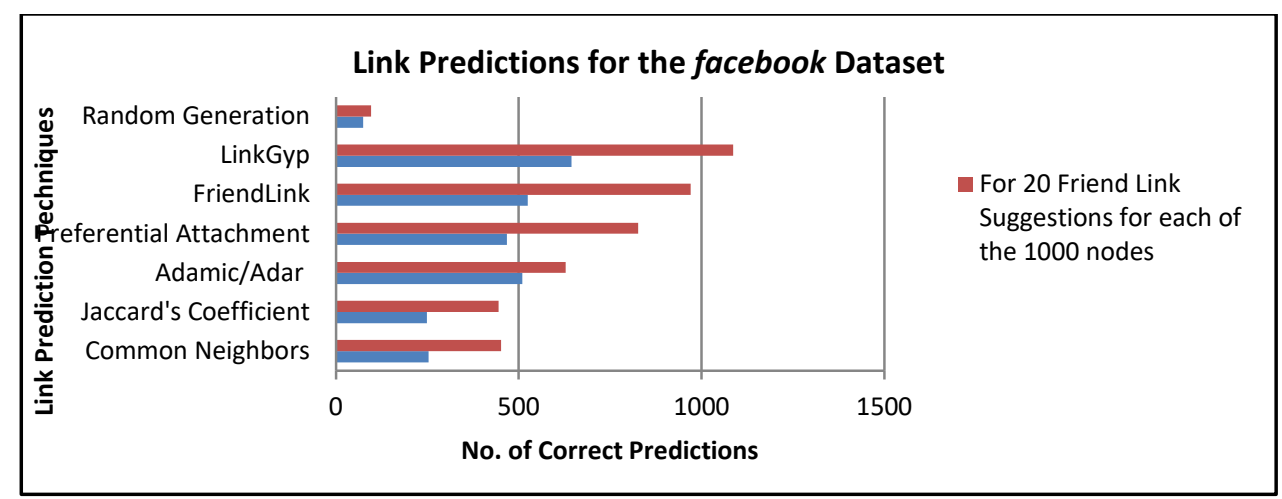

Fig. 5. Number of correct link predictions made on the facebook dataset.

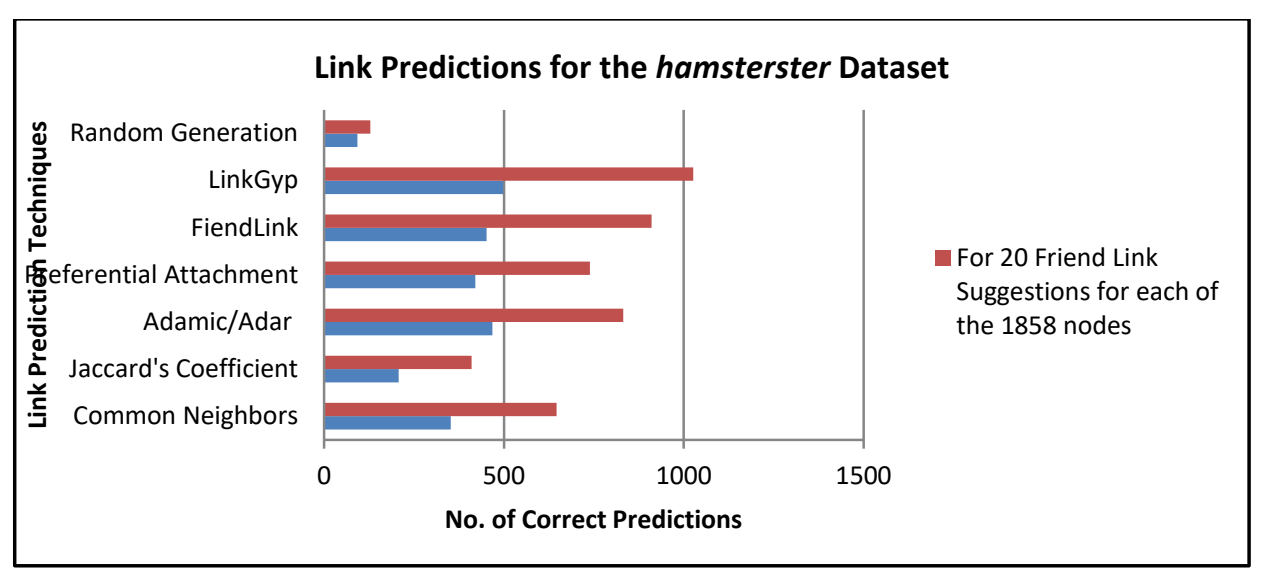

Fig. 6. Number of correct link predictions made on the hamsterster dataset.

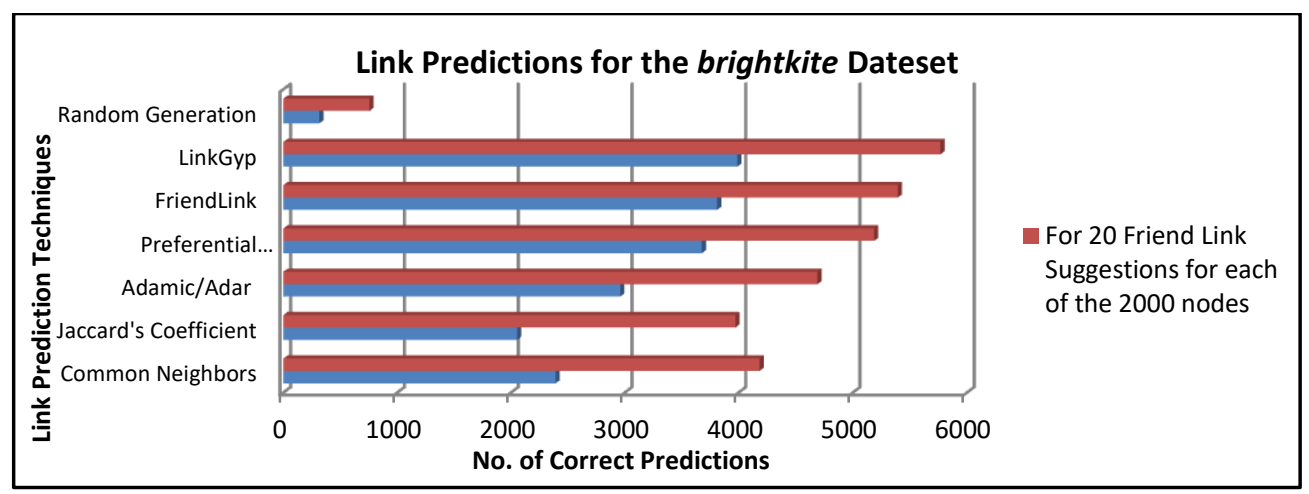

Fig. 7. Number of correct link predictions made on the brightkite dataset. 


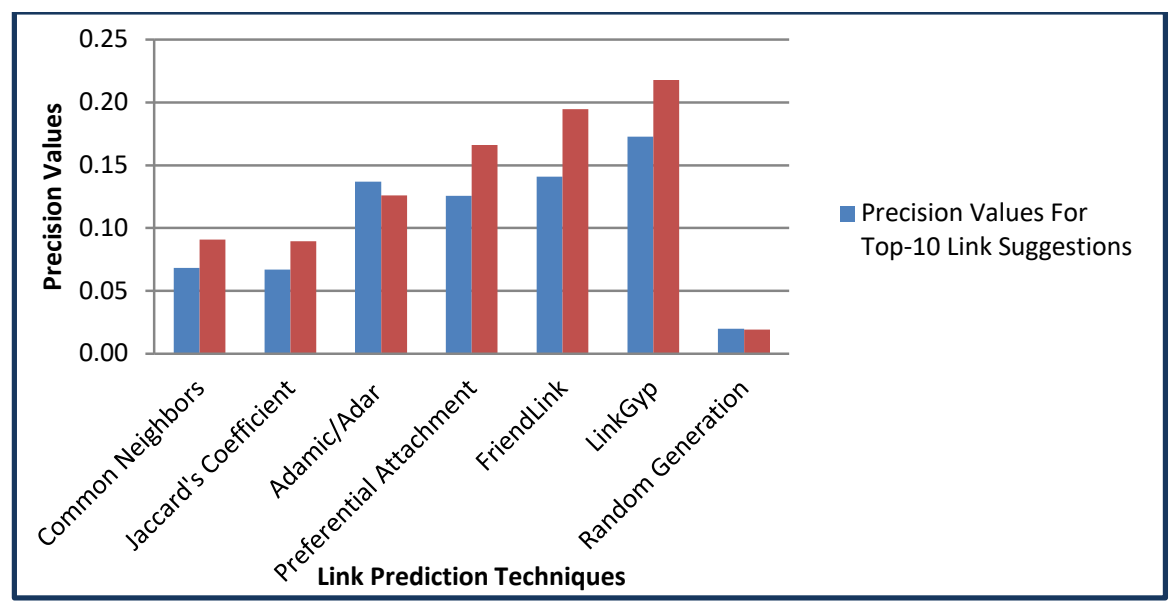

Fig. 8. Precision values of various link prediction techniques for the facebook dataset.

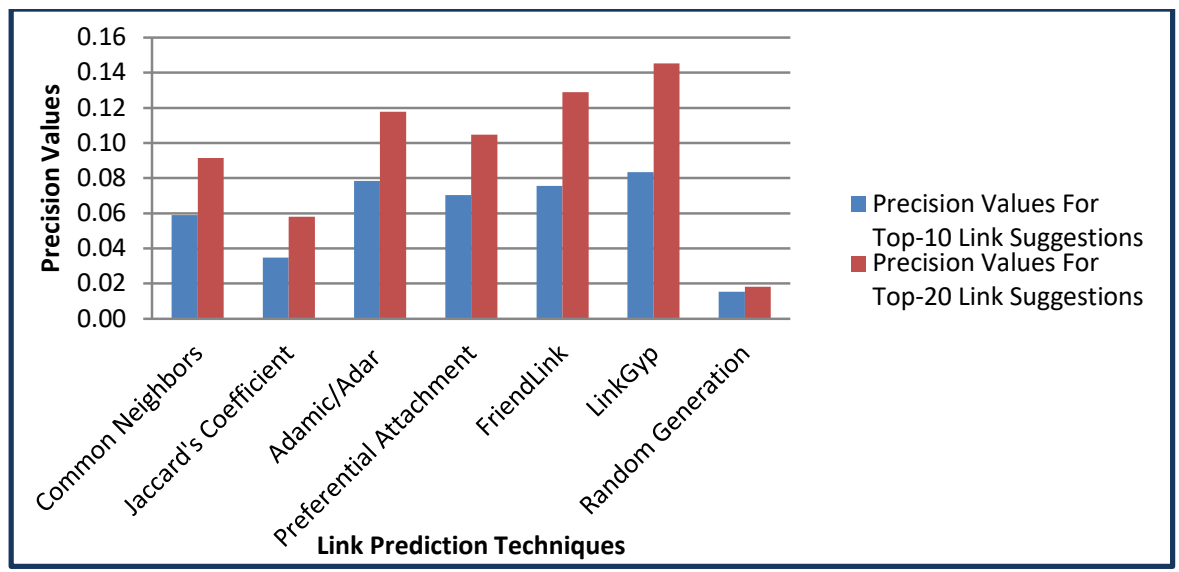

Fig. 9. Precision values of various link prediction techniques for the hamsterster dataset.

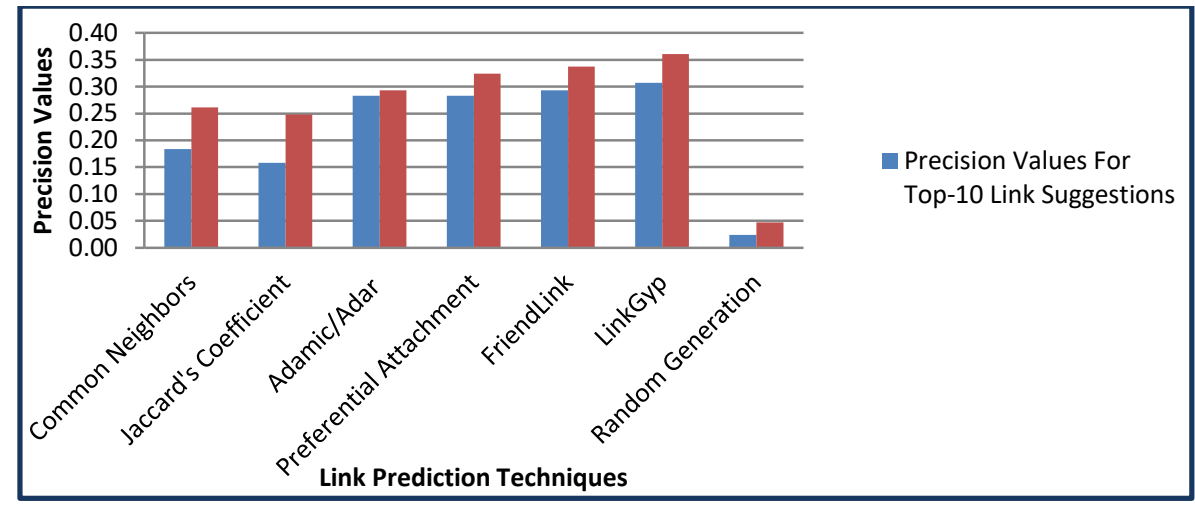

Fig. 10. Precision values of various link prediction techniques for the brightkite dataset.

Usually, for quantifying the accuracy of link prediction techniques, two standard metrics are commonly used: area under the receiver operating characteristic curve (AUC) and Precision [22]. Precision for a link prediction algorithm is calculated by considering the ratio of correct links selected to the total number of links selected. For example, if prediction of two new links has been made for a particular user, out of which one is correct and the other is incorrect, prediction value will be 0.5 . This indicates that higher the precision value, higher will be the prediction accuracy. In this paper, we have used precision as the metric for evaluation of all the link prediction techniques and the results for the three different datasets are given in Fig. 8-10. The results of each of these figures below take into consideration the predictions made for 1000 random distinct nodes present in the facebook dataset, and for each of the 1858 distinct nodes present in the hamsterster dataset, as well as for 2000 distinct nodes present in the brightkite dataset.

\section{CONCLUSIONS AND FUtURE WORK}

The tremendous growth in the use of online social networking sites has forced the researchers to carry out indepth studies in social network mining. The link prediction 
technique in social networks is one such important research area that is in constant focus and is being studied and analyzed for better results. Our proposed work in this paper related to the proposed technique can be summarized as follows:

- This paper initially discusses the five basic standard techniques of link prediction and then gives a comparative analysis of these techniques using experimental results for the same. It can be concluded that these techniques will remain the simplest and basic techniques for studying and analyzing the concept of link prediction for OSNs and can assist a researcher in this field to get a preliminary idea about the same.

- The paper also discusses a new technique of link prediction namely 'LinkGyp' that aims to provide a significantly better result in terms of more correct link predictions among non-linked nodes. We performed several extensive experiments on three different realtime datasets (Facebook, brightkite, and hamsterster) to arrive at a common result which proves that the 'LinkGyp' technique can prove more efficient in prediction of links in social networks compared to several existing approaches.

- Considering link prediction to be one of the key research areas in social network mining, we have made an attempt to further improve the efficiency of link prediction with relate to number of correct predictions as well as run-time complexity.

- Finally, we can conclude that the proposed 'LinkGyp' technique can be considered as the base model for link prediction technique to further carry out experiments on link predictions for complex networks.

As a future work, we plan to study other features of nodes along with their structural properties for generating better and more accurate results for link prediction in social networks. Also, further directions of study are needed to be carried out to improve the algorithm in order to deal with edges having negative weights (signed networks). The proposed algorithm can also be further enhanced to study the cold-start issue and link prediction for signed networks. If all these mentioned issues can also be considered while developing the link prediction techniques, it will provide new insight for modeling prediction of links in social networks.

\section{REFERENCES}

[1] David Liben-Nowell, Jon Kleinberg, The link prediction problem for social networks, Proceedings of the twelfth international conference on Information and knowledge management, (2003), New Orleans, LA, USA [doi 10.1145/956863.956972]

[2] David Liben-Nowell, Jon Kleinberg, The link prediction problem for social networks, Proceedings of the twelfth international conference on Information and knowledge management, (2003), New Orleans, LA, USA [doi 10.1145/956863.956972]

[3] M. Hasan, V. Chaoji, S. Salem, and M. Zaki. Link prediction using supervised learning. In Proceedings of the Workshop on Link Discovery: Issues, Approaches and Applications, (2005)

[4] Elena Zheleva, Lise Getoor, Jennifer Golbeck, Ugur Kuter, Using friendship ties and family circles for link prediction, Proceedings of the
Second international conference on Advances in social network mining and analysis, p.97-113, (2008), Las Vegas, NV, USA

[5] Jilin Chen, Werner Geyer, Casey Dugan , Michael Muller, Ido Guy, Make new friends, but keep the old: recommending people on social networking sites, Proceedings of the SIGCHI Conference on Human Factors in Computing Systems, (2009), Boston, MA, USA [doi>10.1145/1518701.1518735]

[6] Rossano Schifanella, Alain Barrat, Ciro Cattuto, Benjamin Markines, Filippo Menczer, Folks in Folksonomies: social link prediction from shared metadata, Proceedings of the third ACM international conference on Web search and data mining, (2010), New York, New York, USA [doi 10.1145/1718487.1718521]

[7] Papadimitriou, P. Symeonidis, and Y. Manolopoulos, "Fast and accurate link prediction in social networking systems", The Journal of Systems and Software 85, (2012), pp. 2119-2132

[8] M. E. J. Newman, Clustering and preferential attachment in growing networks. Physical Review Letters E, (2001)

[9] Gerard Salton and Michael J. McGill, Introduction to Modern Information Retrieval. McGraw-Hill, (1983).

[10] Lada A. Adamic and Eytan Adar. Friends and neighbors on the web, Social Networks, 25(3):211, (2003).

[11] Jérôme Kunegis, Marcel Blattner, Christine Moser, "Preferential attachment in online networks: measurement and explanations", in Proceedings of the 5th Annual ACM Web Science Conference, pp. 205214, (2013), Paris, France [doi>10.1145/2464464.2464514]

[12] Hamsterster friendships network dataset - KONECT, Available at http://konect.uni-koblenz.de/networks/petster-friendships-hamster, accessed June 2015.

[13] Leo Katz. A new status index derived from sociometric analysis. Psychometrika, 18(1), pp. 39-43, (1953).

[14] Glen Jeh and Jennifer Widom. SimRank: A measure of structuralcontext similarity. In Proceedings of the ACM SIGKDD International Conference on Knowledge Discovery and Data Mining, July (2002).

[15] J. Tang, S. Chang, C. Aggarwal, and H. Liu, "Negative link prediction in social media", In WSDM 2015 - Proceedings of the 8th ACM International Conference on Web Search and Data Mining, pp. 87-96, ACM (2015).

[16] J. Tang, C. Yi, C. Aggarwal, and H. Liu, "A Survey of Signed Network Mining in Social Media”, arXiv preprint arXiv:1511.07569 (2015).

[17] B. Ouyang, L. Jiang and Z. Teng, "A Noise-Filtering Method for Link Prediction in Complex Networks", DOI: 10.1371/journal.pone.0146925 (2016).

[18] F. Liu, B. Liu, C. Sun, M. Liu and X. Wang, "Deep Belief NetworkBased Approaches for Link Prediction in Signed Social Networks", Entropy 17.4, pp. 2140-2169; doi:10.3390/e17042140 (2015).

[19] S. H. Shalforoushan and M. Jalali, "Link prediction in social networks using Bayesian networks", in IEEE International Conference on Artificial Intelligence and Signal Processing, pp. 246-250 (2015).

[20] B. Zhang, S. Choudhury, M. A. Hasan, X. Ning, K. Agarwal, S. Purohit, and P. P. Cabrera "Trust from the past: Bayesian Personalized Ranking based Link Prediction in Knowledge Graphs", in SDM Workshop on Mining Networks and Graphs - MNG 2016, arXiv: 1601.03778, (2016).

[21] E. Cho, S. A. Myers, J. Leskovec. Friendship and Mobility: Friendship and Mobility: User Movement in Location-Based Social Networks, in ACM SIGKDD International Conference on Knowledge Discovery and Data Mining (KDD), 2011.

[22] $\mathrm{L} \mathrm{Lu}$ and $\mathrm{T}$ Zhou, Link prediction in complex networks: A survey. Physica A: Statistical Mechanics and its Applications, 390: 1150-1170, 2011.

[23] R.-H. Li, J. X. Yu, and J. Liu, "Link prediction: the power of maximal entropy random walk, " in CIKM, 2011

[24] Facebook friendships network dataset - http://konect.unikoblenz.de/networks/facebook-wosn-links, accessed June 2016 